\title{
Symptoms and Quality of Life in Patients with Fabry Disease: Results from an International Patient Survey
}

\author{
Olivier Morand $\cdot$ Jack Johnson $\cdot$ Jerry Walter $\cdot$ Leone Atkinson · \\ Gregory Kline $\cdot$ Aline Frey $\cdot$ Juan Politei $\cdot$ Raphael Schiffmann
}

Received: July 17, 2019 / Published online: August 21, 2019

(C) The Author(s) 2019

\begin{abstract}
Introduction: Fabry disease is an X-linked lysosomal storage disorder caused by a deficiency of $\alpha$-galactosidase A. Symptoms include neuropathic pain and gastrointestinal problems, such as diarrhoea. To inform and support the design of a Phase III clinical trial for a new oral treatment for Fabry disease, this study evaluated patients' experiences of Fabry disease symptoms, the impact of symptoms on their quality of life, and their views on participating in clinical trials.
\end{abstract}

Olivier Morand is formerly of Idorsia Pharmaceuticals Ltd, Allschwil, Switzerland.

Enhanced Digital Features To view enhanced digital features for this article go to https://doi.org/10.6084/m9. figshare.9255719.

Electronic supplementary material The online version of this article (https://doi.org/10.1007/s12325-

019-01061-x) contains supplementary material, which is available to authorized users.

O. Morand

Azafaros B.V., Leiden, The Netherlands

J. Johnson

Fabry Support \& Information Group, Concordia,

MO, USA

J. Walter

National Fabry Disease Foundation, Washington,

DC, USA

L. Atkinson - G. Kline

Covance Inc., Princeton, NJ, USA
Methods: An online survey questionnaire was distributed to patients with Fabry disease, through relevant patient organisations. The questionnaire consisted mainly of quantitative, closed questions with pre-defined response options. Fabry-specific pain intensity and its impact on quality of life were rated on a scale from 0 to 10 .

Results: In total, 367 patients completed the survey, of whom half reported frequent pain, moderate to severe pain, and pain in their hands and feet. Pain frequency, intensity and location were similar for males and females. There was no clear association between Fabryspecific pain and the use of enzyme replacement therapy (ERT), with moderate to severe pain reported by $80.4 \%$ of participants receiving ERT and by $75.0 \%$ of participants not receiving ERT. Of participants who were receiving ERT, $35.7 \%$ said they were willing to discontinue it to take part in a clinical trial testing a new oral drug for treating Fabry disease. Gastrointestinal

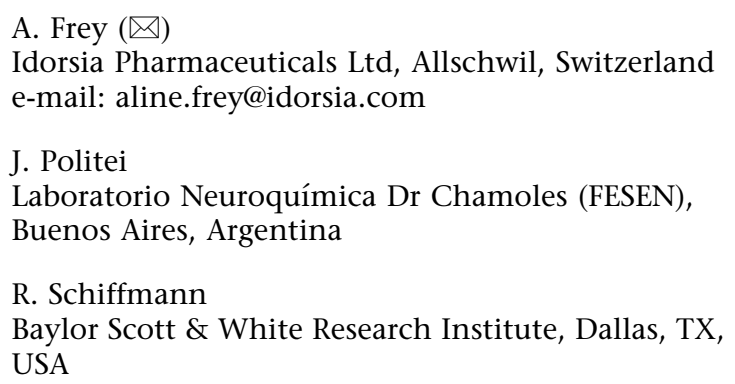


symptoms were more heterogeneous in nature and frequency than Fabry-specific pain, but still affected a significant proportion of participants. Conclusions: Both male and female patients with Fabry disease experience significant Fabryspecific pain, which affects their quality of life. Furthermore, frequent diarrhoea affects many patients. The symptoms occur independently of the use of ERT. This suggests the healthcare needs of patients with Fabry disease are not being fully met, and additional treatments are required to improve symptoms and quality of life.

Funding: This study was sponsored by Actelion Pharmaceuticals Ltd. Study sponsorship was transferred to Idorsia Pharmaceuticals Ltd in July 2018.

Keywords: Clinical trial participation; Fabry disease; Fabry-specific pain; Gastrointestinal symptoms; Genetic disease; Patient survey; Quality of life; Treatment

\section{INTRODUCTION}

Fabry disease is a rare, X-linked lysosomal storage disorder caused by a partial or total deficiency of the enzyme $\alpha$-galactosidase A [1, 2]. As a result, globotriaosylceramide (Gb3) accumulates in cells throughout the body, altering their function. This can cause clinical complications, particularly in the kidneys, heart and nervous system [1, 2].

Fabry disease affects both male hemizygotes and female heterozygotes, although females tend to develop symptoms later and more variably than men [1]. Two phenotypes with differing disease courses have been defined: classical and non-classical Fabry disease [3]. Men with the classical phenotype are more likely to develop Fabry-related complications than men with the non-classical phenotype or women with either phenotype. Men with the non-classical phenotype and women with the classical phenotype have a similar disease course, and women with the classical phenotype are more likely to develop Fabry-related complications than women with the non-classical phenotype [3].
The reported incidence of Fabry disease varies depending on whether the calculation includes patients with non-classical Fabry disease. A study of enzymatic diagnoses estimated that 1 in 117,000 newborns are affected [4]. However, newborn screening studies suggest that, when all phenotypes are accounted for, the incidence of Fabry disease is higher [5-11].

Early Fabry disease symptoms include neuropathic pain and gastrointestinal symptoms. In addition to these symptoms, as the disease progresses, some patients may develop renal failure, cardiovascular disease and cerebrovascular complications [12]. The most common cause of death among patients is Fabry-related cardiovascular disease [13]. To try to preserve kidney and heart function and blood supply to the brain, patients may be treated with enzyme replacement therapy (ERT). Some clinical trials suggest that ERT improves pain and cardiac mass, and slows the decline in kidney function [14-17]. A recent Cochrane review of nine clinical trials concluded that the long-term influence of ERT on the risk of morbidity and mortality related to Fabry disease remains to be established [18].

A pharmacological chaperone, migalastat $\left(\right.$ Galafold $\left.^{\mathrm{TM}}\right)$, has been approved in the European Union, the USA and several other countries as an enzyme enhancement therapy for Fabry disease in patients with amenable mutations [19]. Migalastat functions by stabilising the mutant forms of $\alpha$-galactosidase A, so that the enzyme can be transported to lysosomes to clear the accumulation of Gb3 [20]. According to its Summary of Product Characteristics, approximately $30 \%$ of the 1,014 Fabry disease mutations tested so far are amenable to treatment with migalastat [21].

Another treatment modality being investigated for Fabry disease is substrate reduction therapy (SRT). Lucerastat, an orally available iminosugar, is a potential SRT that inhibits glucosylceramide synthase, an early-step enzyme involved in the synthesis of complex glycosphingolipids, such as Gb3 [22]. A Phase III clinical trial is currently investigating the effect of lucerastat monotherapy on neuropathic pain in patients with Fabry disease of all genotypes (NCT03425539) [23]. As the outcome measures 
of this trial rely on patient-reported outcomes, understanding more about patients' experience of Fabry disease was important for the design of this trial; hence, this patient survey was implemented in parallel with protocol development, and the survey results were used to inform protocol finalisation. The primary objective of the survey was to investigate the intensity, location and frequency of Fabry-specific pain, the frequency and type of gastrointestinal symptoms, the variability of symptoms with/ without ERT use, and the impact of symptoms on quality of life. A secondary objective was to gather information on patients' views about participating in clinical trials for treatments for Fabry disease, and to identify potential barriers to clinical trial enrolment.

\section{METHODS}

\section{Study Design and Patient Selection}

The study was an international, voluntary, cross-sectional, web-based survey that was outsourced to Covance, Princeton, NJ, US and managed by a third party, Schlesinger Associates, Woodbridge, NJ, US. The link to the online survey questionnaire was initially sent via email on 14 November 2016 to all 30 patients with Fabry disease in Schlesinger Associates's inhouse database. It was then distributed by the Fabry International Network, the Fabry Support \& Information Group and the National Fabry Disease Foundation via social media and email to all members in January 2017. All emails and the survey questionnaire clearly stated the purpose of the research, the industry sponsor and information on the collection of personal data. Participation in the survey implied consent. The survey was closed to data collection on 10 July 2017.

The survey questionnaire was available in Chinese (simplified), Dutch, English, French, German, Italian, Korean, Polish, Portuguese, Russian, Spanish (European and Mexican), Taiwanese and Turkish. Translations were conducted via a reputable third-party translation company, and in-country spot checking was carried out to assure flow and readability, and to minimise the potential for in-country inconsistencies. The eligible study population consisted of patients aged 18 years or older with Fabry disease, with access to the internet and with literacy in one of the languages in which the questionnaire was provided. All patients who met these entry criteria and completed the questionnaire were included in the analysis. Their responses to the questions were anonymous.

\section{Compliance with Ethics Guidelines}

Informed consent regarding participation and the use and storage of data was obtained through the survey web form. The data collection tool was compliant with the Health Insurance Portability and Accountability Act and General Data Protection Regulation. Any personal information was fully blinded, and the survey was designed with opt-out mechanisms, to give respondents additional anonymity avenues. The survey questions were developed in conjunction with the Fabry International Network, the Fabry Support \& Information Group and the National Fabry Disease Foundation, with consideration of the core principles of research ethics. The research was classed as low risk by Actelion (initial study sponsor) management, Covance and the patient organisations because of its opt-in survey nature; hence, formal ethical approval by an institutional review board was deemed unnecessary.

\section{Assessments}

The survey questions were developed in conjunction with the Fabry International Network, the Fabry Support \& Information Group and the National Fabry Disease Foundation. The set of questions mainly consisted of quantitative, closed questions with pre-defined response options. A few questions asked participants to provide qualitative information in free-text fields. Only data from quantitative questions are reported in this paper.

The questionnaire began with questions on demographics, including age, sex, country of residence and clinical diagnosis details. 
Participants were then questioned on their views about participating in clinical trials and potential barriers to clinical trial enrolment.

The main body of the questionnaire focussed on the type, intensity, location and frequency of participants' symptoms, in particular Fabryspecific pain, and the impact of these symptoms on their quality of life. Questions on pain intensity and interference with life were structured to align closely with the Brief Pain Inventory (BPI) format, a widely accepted, wellunderstood and validated scale [24]. Initially developed to measure pain in patients with cancer [24], the BPI has also been used to measure pain related to other diseases, including Fabry disease $[2,12,15,17,18,25]$. Fabryspecific pain intensity was rated on a scale from 0 (no pain) to 10 (pain as bad as you could imagine). Pain with an intensity rating of $0-3$ was defined as none to mild, while a rating of 4-7 was defined as moderate and 8-10 was defined as severe. Fabry-specific pain was defined as frequent if it occurred consistently every day; daily, but with varying severity throughout the day; consistently throughout most of the week, with 1-2 days relief; or, if it was at its worst, 1-2 days per week, but otherwise manageable. The interference of pain with quality of life in the 7 days prior to survey completion was rated on a scale from 0 (no interference) to 10 (complete interference). The survey assessed seven domains of quality of life: (1) daily activities, (2) overall mood, (3) walking ability, (4) normal work activities, (5) relationships with other people, (6) sleep patterns and (7) enjoyment of life. Gastrointestinal symptoms (diarrhoea, constipation, early satiety, bloating, heartburn, nausea and vomiting) were defined as frequent if they occurred on at least 6 days per month. The survey concluded with questions about participants' use of ERT. The full list of survey questions is available as Online Resource 1.

\section{Data Analysis}

Results are presented as absolute numbers and percentages. The relationships between variables were analysed by cross-tabulation.

\section{RESULTS}

\section{Demographics and Clinical Characteristics}

In total, 367 completed questionnaires were received. The demographic characteristics of the survey participants are shown in Table 1.

\section{Fabry-Specific Pain}

Fabry-specific pain intensity, location and frequency are shown in Table 2. When asked to consider their Fabry-specific pain at its most severe, 79.3\% (291/367) of participants reported it as moderate or severe. Pain in the hands and feet was common, with $84.2 \%(309 / 367)$ of participants reporting it, irrespective of abdominal pain.

More than half of the participants $(54.2 \%$, 199/367) reported frequent Fabry-specific pain.

Cross-tabulation of the variables showed that $51.5 \%(189 / 367)$ of participants reported frequent pain and moderate to severe pain; $52.0 \%$ (191/367) reported frequent pain and pain in their hands and feet; and $74.7 \%$ (274/367) reported pain in their hands and feet, and moderate to severe pain. Fifty percent of participants $(183 / 367)$ reported all three domains: frequent pain, moderate to severe pain, and pain in their hands and feet.

\section{Fabry-Specific Pain by Sex}

Pain intensity was comparable between males and females: $77.6 \%(190 / 245)$ of females reported moderate to severe pain compared with $82.5 \%(99 / 120)$ of males (Table 2). The location and frequency of pain were also comparable between the sexes: $81.6 \%(200 / 245)$ of females and $89.2 \%(107 / 120)$ of males reported pain in their hands and feet, and $52.2 \%(128 / 245)$ of females and $59.2 \%(71 / 120)$ of males reported frequent pain (Table 2 ).

\section{Fabry-Specific Pain by Age}

The proportion of participants who reported moderate to severe pain decreased with age (Fig. 1). A similar trend was seen for pain in the 
Table 1 Demographic characteristics of Fabry disease survey participants, all and by sex

\begin{tabular}{|c|c|c|c|c|c|c|}
\hline \multirow[t]{2}{*}{ Characteristic } & \multicolumn{2}{|c|}{ All $(n=367)$} & \multicolumn{2}{|c|}{ Male $(n=120)$} & \multicolumn{2}{|c|}{ Female $(n=245)$} \\
\hline & $\bar{n}$ & $\%$ & $n$ & $\%$ & $n$ & $\%$ \\
\hline \multicolumn{7}{|l|}{ Age group, years } \\
\hline $18-40$ & 126 & 34.3 & 40 & 33.3 & 84 & 34.3 \\
\hline $41-60$ & 195 & 53.1 & 72 & 60.0 & 123 & 50.2 \\
\hline $61-80$ & 46 & 12.5 & 8 & 6.7 & 38 & 15.5 \\
\hline \multicolumn{7}{|l|}{ Country of residence } \\
\hline USA & 200 & 54.5 & 57 & 47.5 & 142 & 58.0 \\
\hline Canada & 47 & 12.8 & 19 & 15.8 & 28 & 11.4 \\
\hline Other $^{\mathrm{a}}$ & 120 & 32.7 & 44 & 36.7 & 75 & 30.6 \\
\hline \multicolumn{7}{|c|}{ Diagnosis confirmed by genetic testing } \\
\hline Yes & 348 & 94.8 & 114 & 95.0 & 233 & 95.1 \\
\hline No & 5 & 1.4 & 2 & 1.7 & 2 & 0.8 \\
\hline Not sure & 14 & 3.8 & 4 & 3.3 & 10 & 4.1 \\
\hline \multicolumn{7}{|l|}{ Time since diagnosis, months } \\
\hline$<12$ & 29 & 7.9 & 4 & 3.3 & 25 & 10.2 \\
\hline $12-24$ & 18 & 4.9 & 4 & 3.3 & 14 & 5.7 \\
\hline$>24$ & 320 & 87.2 & 112 & 93.3 & 206 & 84.1 \\
\hline \multicolumn{7}{|l|}{ Current ERT use } \\
\hline Yes & 280 & 76.3 & 105 & 87.5 & 174 & 71.0 \\
\hline No, never tried it & 66 & 18.0 & 7 & 5.8 & 58 & 23.7 \\
\hline No, tried it, no longer on it & 18 & 4.9 & 7 & 5.8 & 11 & 4.5 \\
\hline Not sure & 3 & 0.8 & 1 & 0.8 & 2 & 0.8 \\
\hline
\end{tabular}

Two participants who preferred not to indicate their sex were not included in the per-sex analysis

$E R T$ enzyme replacement therapy

a Other includes France $(n=1)$, Germany $(n=1)$, Italy $(n=1)$, the Netherlands $(n=1)$, Poland $(n=18)$, Portugal $(n=12)$, Russia $(n=1)$, Spain $(n=1)$, Turkey $(n=2)$, the United Kingdom $(n=43)$ and other countries not listed in the survey $(n=39)$

hands and feet, which declined from 89.7\% (113/ 126) of participants aged $18-40$ years to $76.1 \%$ $(35 / 46)$ of participants aged over 60 years (Online Resource 2). However, increasing age had little effect on pain frequency (Online Resource 3).

\section{Fabry-Specific Pain by ERT Use}

Participants receiving ERT and participants not receiving ERT were similarly affected by Fabry- specific pain (Table 3 ). Moderate to severe pain was reported by $80.4 \%(225 / 280)$ of participants receiving ERT and by $75.0 \%(63 / 84)$ of participants not receiving ERT. Pain in the hands and feet was reported by $83.6 \%(234 / 280)$ of participants receiving ERT and by $85.7 \%(72 / 84)$ of participants not receiving ERT. 
Table 2 Intensity, location and frequency of Fabry-specific pain, in all participants and by sex

\begin{tabular}{|c|c|c|c|c|c|c|}
\hline \multirow[t]{2}{*}{ Pain domain } & \multicolumn{2}{|c|}{ All $(n=367)$} & \multicolumn{2}{|c|}{ Male $(n=120)$} & \multicolumn{2}{|c|}{ Female $(n=245)$} \\
\hline & $\bar{n}$ & $\%$ & $\bar{n}$ & $\%$ & $\bar{n}$ & $\%$ \\
\hline \multicolumn{7}{|l|}{ Intensity ${ }^{\mathrm{a}}$} \\
\hline None to mild & 76 & 20.7 & 21 & 17.5 & 55 & 22.4 \\
\hline Moderate & 159 & 43.3 & 53 & 44.2 & 105 & 42.9 \\
\hline Severe & 132 & 36.0 & 46 & 38.3 & 85 & 34.7 \\
\hline \multicolumn{7}{|l|}{ Location } \\
\hline Hands and feet with abdominal pain & 223 & 60.8 & 76 & 63.3 & 145 & 59.2 \\
\hline Hands and feet without abdominal pain & 86 & 23.4 & 31 & 25.8 & 55 & 22.4 \\
\hline No pain in hands and feet with or without abdominal pain & 58 & 15.8 & 13 & 10.8 & 45 & 18.4 \\
\hline \multicolumn{7}{|l|}{ Frequency } \\
\hline Frequent $^{\mathrm{b}}$ & 199 & 54.2 & 71 & 59.2 & 128 & 52.2 \\
\hline Once or twice per month & 86 & 23.4 & 27 & 22.5 & 58 & 23.7 \\
\hline Once or twice per year & 23 & 6.3 & 4 & 3.3 & 18 & 7.3 \\
\hline No pain & 30 & 8.2 & 7 & 5.8 & 23 & 9.4 \\
\hline Other & 29 & 7.9 & 11 & 9.2 & 18 & 7.3 \\
\hline
\end{tabular}

Two participants who preferred not to indicate their sex were not included in the per-sex analysis

a Pain intensity ratings (scale $0-10$ ): none to mild: $0-3$; moderate: 4-7; severe: $8-10$

b Frequent pain was defined as (1) consistent pain every day, (2) daily pain that varied in severity throughout the day, (3) consistent pain throughout most of the week with 1-2 days relief, or (4) pain at its worst 1-2 days per week, but otherwise manageable

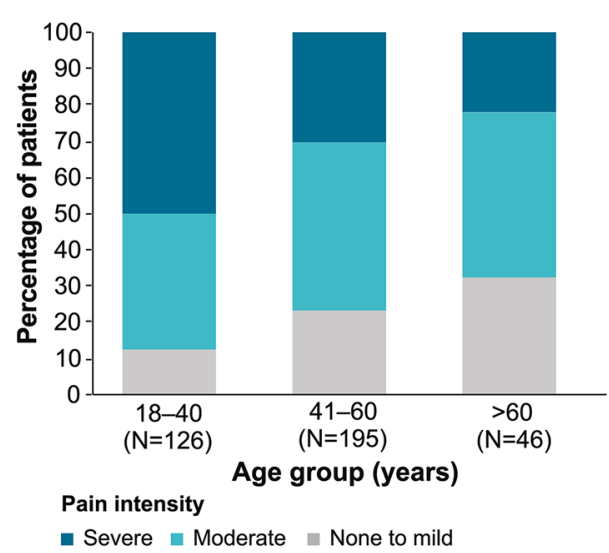

Fig. 1 Intensity of pain by age; pain intensity ratings (scale 0-10): none to mild: $0-3$; moderate: $4-7$; severe: $8-10$

\section{Effect of Pain on Quality of Life}

Participants who were minimally impacted by Fabry-specific pain [(i.e. those who reported inconsistent and infrequent pain $(n=168)$ ] were not prompted to provide further information, and are therefore not included in the quality-of-life analyses. For the 199 participants who reported consistent and frequent pain, Fig. 2 shows the cross-tabulation of pain intensity and interference with the various qualityof-life domains. For all quality-of-life domains, the level of interference increased with increasing pain intensity.

\section{Gastrointestinal Symptoms}

The frequency of gastrointestinal symptoms according to sex is shown in Table 4. Frequent 
Table 3 Intensity, location and frequency of Fabry-specific pain in participants receiving ERT and in participants not receiving ERT

\begin{tabular}{|c|c|c|c|c|}
\hline \multirow[t]{2}{*}{ Pain domain } & \multicolumn{2}{|c|}{ Receiving ERT $(n=280)$} & \multicolumn{2}{|c|}{ Not receiving ERT $(n=84)$} \\
\hline & $\bar{n}$ & $\%$ & $n$ & $\%$ \\
\hline \multicolumn{5}{|l|}{ Intensity $^{\mathrm{a}}$} \\
\hline None to mild & 55 & 19.6 & 21 & 25.0 \\
\hline Moderate & 125 & 44.6 & 31 & 36.9 \\
\hline Severe & 100 & 35.7 & 32 & 38.1 \\
\hline \multicolumn{5}{|l|}{ Location } \\
\hline Hands and feet with abdominal pain & 162 & 57.9 & 59 & 70.2 \\
\hline Hands and feet without abdominal pain & 72 & 25.7 & 13 & 15.5 \\
\hline No pain in hands and feet with/without abdominal pain & 46 & 16.4 & 12 & 14.3 \\
\hline \multicolumn{5}{|l|}{ Frequency } \\
\hline Frequent ${ }^{\mathrm{b}}$ & 153 & 54.6 & 43 & 51.2 \\
\hline Once or twice per month & 67 & 23.9 & 19 & 22.6 \\
\hline Once or twice per year & 16 & 5.7 & 7 & 8.3 \\
\hline No pain & 19 & 6.8 & 11 & 13.1 \\
\hline Other & 25 & 8.9 & 4 & 4.8 \\
\hline
\end{tabular}

Three patients who were unsure about their current use of ERT were not included in the analysis

${ }^{a}$ Pain intensity ratings (scale $0-10$ ): none to mild: $0-3$; moderate: $4-7$; severe: $8-10$

b Frequent pain was defined as (1) consistent pain every day, (2) daily pain that varied in severity throughout the day, (3) consistent pain throughout most of the week with 1-2 days relief, or (4) pain at its worst 1-2 days per week, but otherwise manageable

$E R T$ enzyme replacement therapy

diarrhoea was reported by $39.2 \%(47 / 120)$ of males and $23.3 \%(57 / 245)$ of females. The frequency of diarrhoea was comparable between participants receiving ERT $(29.6 \%, 83 / 280)$ and those who were not $(23.8 \%, 20 / 84)$ (Online Resource 4). Frequent constipation was more common in females $(24.5 \%, 60 / 245)$ than in males $(4.2 \%, 5 / 120)$; in females, frequent constipation and frequent diarrhoea were equally as common.

Cross-tabulation of Fabry-specific pain intensity and gastrointestinal symptoms showed that $25.3 \%(93 / 367)$ of participants reported moderate to severe pain and frequent diarrhoea (Online Resource 5). Also, 22.1\% (81/ 367 ) of participants reported abdominal pain and diarrhoea for at least 6 days per month (Online Resource 6).

\section{Participation in Clinical Trials}

Almost one-third of participants (31.3\%, $115 / 367$ ) were participating or had previously participated in a clinical trial. All participants were asked to rank proposed reasons for participating in a clinical trial. All reasons were ranked highly, with $88-98 \%$ of participants ranking them as very or somewhat important (Fig. 3).

Participants were also asked how comfortable they felt about taking part in activities related to clinical trial participation (Online 

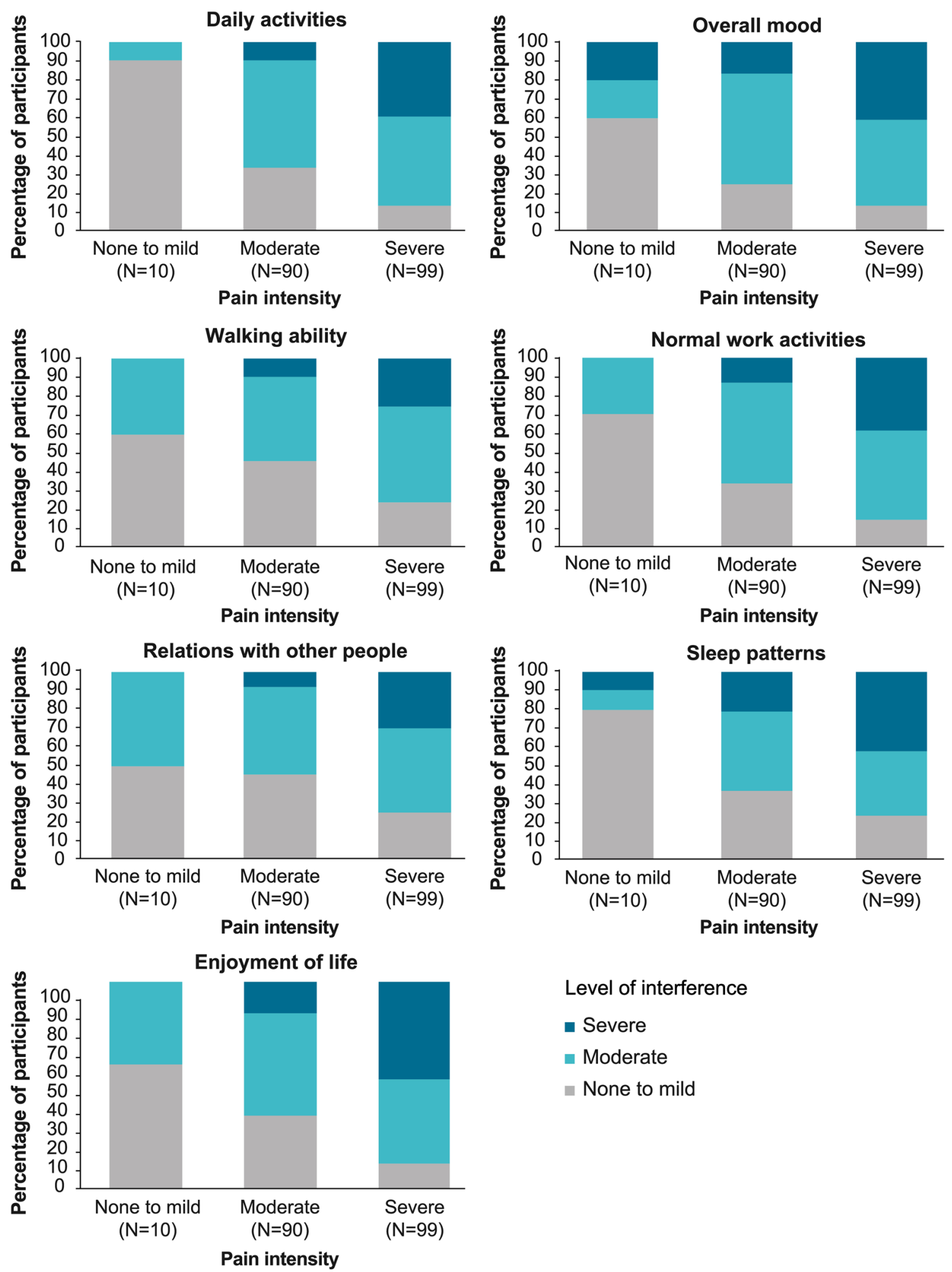

$$
\begin{aligned}
& \text { Level of interference } \\
& \text { Severe } \\
& \text { Moderate } \\
& \text { None to mild }
\end{aligned}
$$

Fig. 2 Interference with seven quality-of-life domains in participants with frequent and consistent pain, by pain intensity; pain intensity/interference ratings (scale $0-10)$ : none to mild: $0-3$; moderate: $4-7$; severe: $8-10$ 
Table 4 Frequency of gastrointestinal symptoms by sex

\begin{tabular}{lccccc}
\hline $\begin{array}{l}\text { Symptoms, } \\
\text { days/month }\end{array}$ & \multicolumn{2}{c}{ Female $(n=245)$} & & \multicolumn{2}{c}{ Male $(n=120)$} \\
\cline { 2 - 3 } \cline { 5 - 6 } Diarrhoea & $n$ & $\%$ & & $n$ & $\%$ \\
\hline 0 & 119 & 48.6 & & 30 & 25.0 \\
$1-5$ & 69 & 28.2 & & 43 & 35.8 \\
$6+$ & 57 & 23.3 & & 47 & 39.2 \\
Constipation & & & & \\
0 & 121 & 49.4 & & 91 & 75.8 \\
$1-5$ & 64 & 26.1 & & 24 & 20.0 \\
$6+$ & 60 & 24.5 & & 5 & 4.2
\end{tabular}

Early satiety$$
0
$$$$
1-5
$$$$
6+
$$$$
153
$$$$
23
$$$$
69
$$

Bloating

0
$1-5$
$6+$

Heartburn

$$
0
$$$$
1-5
$$$$
6+
$$

Nausea

\begin{tabular}{lrrrr}
0 & 144 & 58.8 & 73 & 60.8 \\
$1-5$ & 53 & 21.6 & 26 & 21.7 \\
$6+$ & 48 & 19.6 & 21 & 17.5 \\
Vomiting & & & & \\
0 & 220 & 89.8 & 93 & 77.5 \\
$1-5$ & 16 & 6.5 & 23 & 19.2 \\
$6+$ & 9 & 3.7 & 4 & 3.3 \\
Other & & & & \\
0 & 200 & 81.6 & 107 & 89.2 \\
$1-5$ & 17 & 6.9 & 10 & 8.3 \\
\hline
\end{tabular}

Table 4 continued

\begin{tabular}{|c|c|c|c|c|}
\hline \multirow{2}{*}{$\begin{array}{l}\text { Symptoms, } \\
\text { days/month }\end{array}$} & \multicolumn{2}{|c|}{ Female $(n=245)$} & \multicolumn{2}{|c|}{ Male $(n=120)$} \\
\hline & $n$ & $\%$ & $n$ & $\%$ \\
\hline $6+$ & 28 & 11.4 & 3 & 2.5 \\
\hline
\end{tabular}

Two participants who preferred not to indicate their sex were not included in the analysis $E R T$ enzyme replacement therapy

Resource 7). While most participants (95.4\%, 350/367) felt comfortable about providing standard biological samples, such as blood and urine, only $55.3 \%(203 / 367)$ felt comfortable about providing additional biological samples, for example via a biopsy. For each of the other activities, including radiological procedures, spending nights away from home and switching to an experimental oral therapy, over $70 \%$ of participants reported they felt comfortable.

Over one-third of participants (34.6\%, $127 / 367)$ reported that the main barrier to participating in a trial was potential side effects (Table 5). Other barriers included the time required to participate $(15.3 \%, 56 / 367)$, distance to trial site $(10.1 \%, 37 / 367)$ and uncertainty about the benefits $(7.9 \%, 29 / 367)$.

Over one-third of participants who were receiving ERT $(35.7 \%, 101 / 283)$ said they would be willing to discontinue ERT to take part in a 12-month, blinded, placebo-controlled clinical trial testing a new oral drug for the treatment of Fabry disease. A further $40.3 \%(114 / 283)$ of participants were unsure, and the remaining $24.0 \%(68 / 283)$ were unwilling to discontinue ERT.

\section{DISCUSSION}

This survey concomitantly assessed the three domains of Fabry-specific pain: intensity, location and frequency. It shows that this pain is common in patients with Fabry disease, with half of participants experiencing moderate to severe pain, frequent pain and pain in their hands and feet. Previous studies have shown that Fabry-specific pain has a negative impact 


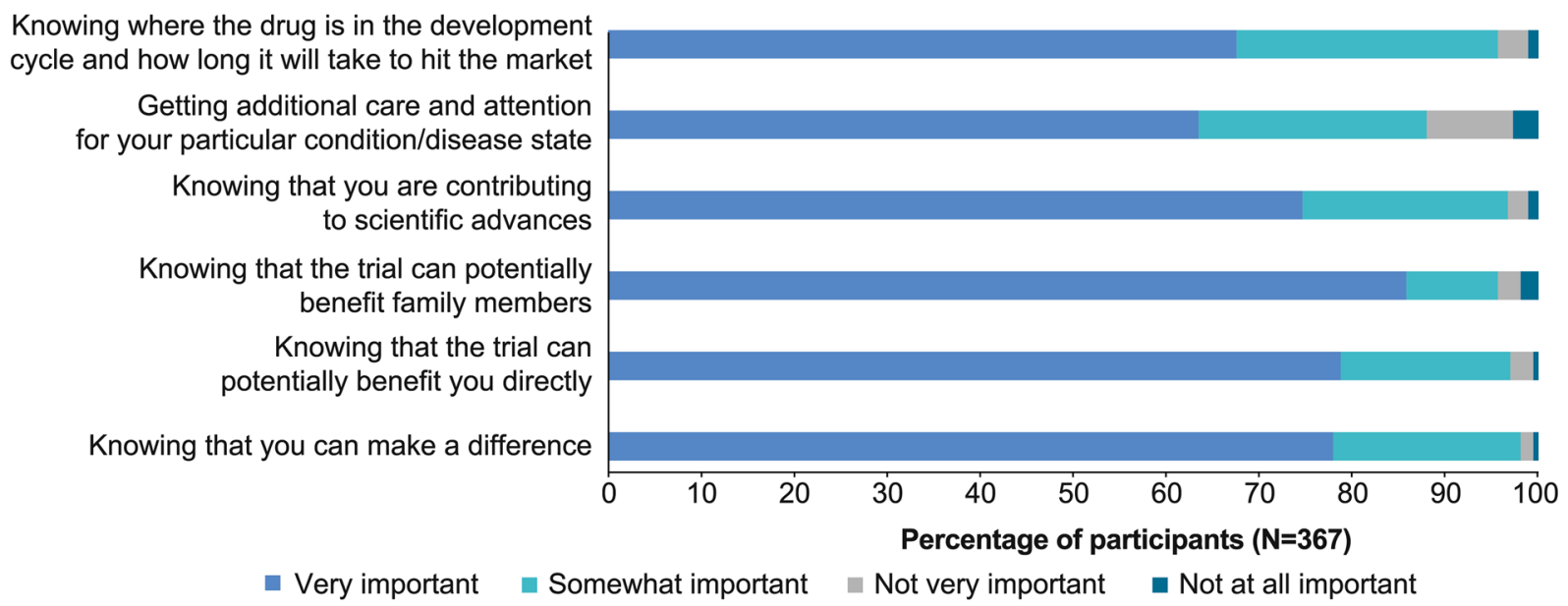

Fig. 3 Reasons for participation in a clinical trial, ranked by importance

Table 5 Barriers to participation in a clinical trial, all and by sex

\begin{tabular}{|c|c|c|c|c|c|c|}
\hline \multirow[t]{2}{*}{ Number one reason preventing participation } & \multicolumn{2}{|c|}{ All $(n=367)$} & \multicolumn{2}{|c|}{ Male $(n=120)$} & \multicolumn{2}{|c|}{ Female $(n=245)$} \\
\hline & $n$ & $\%$ & $n$ & $\%$ & $n$ & $\%$ \\
\hline Potential side effects & 127 & 34.6 & 45 & 37.5 & 82 & 33.5 \\
\hline Time required & 56 & 15.3 & 17 & 14.2 & 38 & 15.5 \\
\hline Distance to trial site & 37 & 10.1 & 12 & 10.0 & 25 & 10.2 \\
\hline Uncertainty regarding the benefit & 29 & 7.9 & 13 & 10.8 & 16 & 6.5 \\
\hline Uncertainty regarding insurance coverage & 26 & 7.1 & 3 & 2.5 & 22 & 9.0 \\
\hline Little awareness of trials & 20 & 5.4 & 8 & 6.7 & 12 & 4.9 \\
\hline Inconvenience & 18 & 4.9 & 7 & 5.8 & 11 & 4.5 \\
\hline Compensation & 6 & 1.6 & 4 & 3.3 & 2 & 0.8 \\
\hline Hearing about someone else's experience & 5 & 1.4 & 0 & 0.0 & 5 & 2.0 \\
\hline Not interested & 2 & 0.5 & 0 & 0.0 & 2 & 0.8 \\
\hline Other & 41 & 11.2 & 11 & 9.2 & 30 & 12.2 \\
\hline
\end{tabular}

Two participants who preferred not to indicate their sex were not included in the per-sex analysis

on quality of life $[2,12,26]$. Similarly, our survey found an association between pain intensity and effect on quality of life, with most of the participants who reported severe pain also reporting moderate to severe interference in all of the seven quality-of-life domains assessed.

Gastrointestinal symptoms are more heterogeneous in nature and frequency than Fabryspecific pain, but still affect a significant proportion of patients. The survey found that twice as many females than males reported constipation at least one day per month. This finding is unlikely to be Fabry-related, instead it is consistent with constipation data for the general population $[27,28]$, in which female-tomale ratios as high as 3.77 have been reported [27]. This sex difference is thought to be related to hormones [27, 28]. Cross-tabulation of 
gastrointestinal symptoms with Fabry-specific pain revealed that nearly one-third of participants with moderate to severe pain have frequent diarrhoea and nearly three-quarters have abdominal pain.

Interestingly, the survey found that a high proportion of participants receiving ERT had Fabry-specific pain. ERT with $\alpha$-galactosidase A has previously been shown to be modestly effective at reducing the severity of neuropathic pain over time, both in a randomised control trial and in the Fabry Outcome Survey $[15,17]$. However, our results support those of a singlecentre, retrospective study in which $84 \%$ of patients reported that ERT had no effect on their pain [29]. The continuing presence of pain in many patients despite ERT suggests that patients need new treatment options for Fabry disease.

Two-thirds of survey participants were female, whereas Fabry registries report roughly equal proportions of males and females [30, 31]. One possible reason for this discrepancy is that women are reported to be more willing than men to participate in health surveys [32].

Despite some reports that neuropathic pain is less prevalent and less severe in women $[2,26,33]$, this survey found that the intensity, location and frequency of pain were comparable between the sexes. This is in line with the results of a smaller survey $(n=88)$ published in 2006, which found that pain intensity and the impairment to quality of life was similar between females and males [26]. The authors of that survey suggested that their results meant that either females have Fabry pain equivalent in intensity to males, or they have less intense pain but are less likely to receive adequate pain medication [26]. This survey could not verify either of these suggestions as it did not assess pain management. However, this survey did find that a higher proportion of females than males had never tried ERT (23.7 vs. 5.8\%), which may suggest that females are either less likely to be offered therapy for Fabry disease or more likely to refuse it.

The intensity of pain appeared to decrease with increasing age. This is consistent with previous studies, which have reported a negative association between pain and disease duration in males [26]. This could be because patients get used to their pain over time, or because progressive damage to nerve fibres over time impairs nerve function, thus the patient feels less pain [26]. Alternatively, the correlation could be related to phenotype: a recent study found that pain was a common first symptom of Fabry disease in patients with classic mutations, but was not reported in patients with the lateonset N215S mutation [34].

This survey suggests that additional treatments are needed to improve symptoms and quality of life in patients with Fabry disease, thus supporting the concept for the ongoing, prospective, multicentre, double-blind, randomised, placebo-controlled, parallel-group Phase III trial with lucerastat monotherapy [23]. Running the survey in parallel to developing the trial protocol helped to consolidate its design and to confirm the choice of primary outcome measure (neuropathic pain). The survey found that the main barriers to patients' participation in such a trial are potential side effects, time required, distance to trial site and uncertainty about the benefits. The survey also investigated the requirement to discontinue ERT as a potential barrier to patient participation, and found that over one-third of survey participants were willing to discontinue ERT to take part in a placebo-controlled trial, and a further $40 \%$ may consider it.

This survey has some limitations. The survey was online and anonymous, so participants could have completed the survey more than once. However, industry best practices for quality control were implemented to minimise the potential for duplication. It was not possible to guarantee that a family member or someone else without Fabry disease did not take the survey. However, screening questions at the start of the survey minimised the risk of this occurring. In addition, the survey was distributed through the most reputable of patient forums, and, upon completion of the research, thorough data review was conducted to eliminate any potential anomalies, such as straight lining. Testing conditions for the validated BPI were not implemented in the direct-to-patient approach. As most participants were from Europe and North America, and participation numbers were 
low in some European countries, the responses may not represent the diversity of cultural specificities of patients with Fabry disease. The study may be affected by recruitment bias: participants took part voluntarily in the survey and may be systematically different from those who did not participate. In addition, participants on ERT may be phenotypically different from those who were not on ERT, which may have affected results. Results may also have been affected by undefined recall periods, and by the understanding and order of questions. Participants were not asked if they had joint pain, an important symptom of Fabry disease that doctors may overlook or misdiagnose [25]. They were also not asked how they manage the pain for their disease, so responses to the pain-related questions may have been affected by the use of pain medication. The survey was structured to capture patients' perspective of their disease and did not define 'Fabry-specific pain'; different patients may have interpreted the term differently. Data were descriptive only and thus the statistical significance of the results is unknown. Despite these limitations, this survey adds valuable insight into Fabry symptoms and their effect on quality of life, which could be used to influence care for these patients. The ongoing lucerastat Phase III trial is expected to help the Fabry community understand further the symptoms of Fabry disease, in particular through the use of a Fabry-specific instrument to measure neuropathic pain.

\section{CONCLUSIONS}

Patients with Fabry disease who participated in this large, international survey experience significant Fabry-specific pain, independent of sex or ERT use. This pain significantly affects their daily activities and quality of life. Although gastrointestinal symptoms are more heterogeneous in nature and frequency than pain, frequent diarrhoea affects a significant proportion of patients (more often male than female), independent of ERT use. These results suggest that the healthcare needs of patients with Fabry disease are not being fully met, and additional treatments are required to improve symptoms and quality of life.

\section{ACKNOWLEDGEMENTS}

The authors would like to thank all the patients who participated in this survey and Schlesinger Associates, Woodbridge, NJ, US, for managing the execution of the survey. The authors dedicate this manuscript to the memory of Christine Lavery, former President of the Fabry International Network. As well as contributing to survey development and distribution for this manuscript, Christine was instrumental in raising awareness of, and support for, many rare inherited diseases over many years.

Funding. This study was sponsored by Actelion Pharmaceuticals Ltd. Study sponsorship was transferred to Idorsia Pharmaceuticals Ltd in July 2018. The Sponsor (initially Actelion Pharmaceuticals Ltd, then Idorsia Pharmaceuticals Ltd) participated in the design and conduct of the study, collection, management, analysis and interpretation of the data. The Sponsor funded the journal's Rapid Service and Open Access Fees. All authors had full access to all of the data in this study and take complete responsibility for the integrity of the data and accuracy of the data analysis.

Medical Writing and/or Editorial Assistance. The authors would like to thank Joanne Redfern, MChem, of InterComm International Ltd, Cambridge, UK, for providing medical writing support, which was funded by Idorsia Pharmaceuticals Ltd, Allschwil, Switzerland, in accordance with Good Publication Practice (GPP3) guidelines (http://www.ismpp.org/ gpp3).

Authorship. All named authors meet the International Committee of Medical Journal Editors (ICMJE) criteria for authorship for this article, take responsibility for the integrity of the work as a whole, and have given their approval for this version to be published. 
Author Contributions. OM and AF contributed to the survey design, and generated, analysed and interpreted the results. JJ and JW contributed to the survey design and distributed the survey to participants. LA and GK contributed to the survey design, executed the survey, set up the survey database and generated, analysed and interpreted the results. JP and RS analysed and interpreted the results. All authors were involved in drawing conclusions from the results.

Disclosures. OM received a salary as an employee of Idorsia and holds Idorsia stock. JJ is part of the Fabry Support \& Information Group, which has received an unrestricted grant from Idorsia. RS received grant support from Shire/ Takeda, and travel and honoraria from Amicus Therapeutics and Protalix Biotherapeutics for Fabry disease-related work. AF receives a salary as an employee of Idorsia, and holds Idorsia stock and share options. JW, LA, GK and JP have nothing to declare.

Compliance with Ethics Guidelines. Informed consent regarding participation and the use and storage of data was obtained through the survey web form. The data collection tool was compliant with the Health Insurance Portability and Accountability Act and General Data Protection Regulation. Any personal information was fully blinded, and the survey was designed with opt-out mechanisms, to give respondents additional anonymity avenues. The survey questions were developed in conjunction with the Fabry International Network, the Fabry Support \& Information Group and the National Fabry Disease Foundation, with consideration of the core principles of research ethics. The research was classed as low-risk by Actelion (initial study sponsor) management, Covance Inc. and the patient organisations because of its opt-in survey nature; hence, formal ethical approval by an institutional review board was deemed unnecessary.

Data Availability. Some of the data generated or analysed during this study are included in this published article and as supplementary information files. Additional datasets generated and/or analysed during the study are available from the corresponding author on reasonable request.

Open Access. This article is distributed under the terms of the Creative Commons Attribution-NonCommercial 4.0 International License (http://creativecommons.org/licenses/ by-nc/4.0/), which permits any noncommercial use, distribution, and reproduction in any medium, provided you give appropriate credit to the original author(s) and the source, provide a link to the Creative Commons license, and indicate if changes were made.

\section{REFERENCES}

1. Giugliani RN, Niu D-M, Ramaswami U, West M, Hughes D, Kampmann C, Pintos-Morell G, et al. A 15-year perspective of the Fabry outcome survey. J Inborn Errors Metab Screen. 2016;4:1-12.

2. Arends M, Korver S, Hughes DA, Mehta A, Hollak CEM, Biegstraaten M. Phenotype, disease severity and pain are major determinants of quality of life in Fabry disease: results from a large multicenter cohort study. J Inherit Metab Dis. 2018;41(1):141-9.

3. Arends M, Wanner C, Hughes D, Mehta A, Oder D, Watkinson OT, et al. Characterization of classical and nonclassical Fabry disease: a multicenter study. J Am Soc Nephrol. 2017;28(5):1631-41.

4. Meikle PJ, Hopwood JJ, Clague AE, Carey WF. Prevalence of lysosomal storage disorders. JAMA. 1999;281(3):249-54.

5. Burton BK, Charrow J, Hoganson GE, Waggoner D, Tinkle B, Braddock SR, et al. Newborn screening for lysosomal storage disorders in Illinois: the initial 15-month experience. J Pediatr. 2017;190:130-5.

6. Hopkins PV, Campbell C, Klug T, Rogers S, RaburnMiller J, Kiesling J. Lysosomal storage disorder screening implementation: findings from the first six months of full population pilot testing in Missouri. J Pediatr. 2015;166(1):172-7.

7. Lin HY, Chong KW, Hsu JH, Yu HC, Shih CC, Huang $\mathrm{CH}$, et al. High incidence of the cardiac variant of Fabry disease revealed by newborn screening in the Taiwan Chinese population. Circ Cardiovasc Genet. 2009;2(5):450-6. 
8. Mechtler TP, Stary S, Metz TF, De Jesus VR, GreberPlatzer S, Pollak A, et al. Neonatal screening for lysosomal storage disorders: feasibility and incidence from a nationwide study in Austria. Lancet. 2012;379(9813):335-41.

9. Spada M, Pagliardini S, Yasuda M, Tukel T, Thiagarajan $G$, Sakuraba $H$, et al. High incidence of later-onset fabry disease revealed by newborn screening. Am J Hum Genet. 2006;79(1):31-40.

10. Inoue T, Hattori K, Ihara K, Ishii A, Nakamura K, Hirose S. Newborn screening for Fabry disease in Japan: prevalence and genotypes of Fabry disease in a pilot study. J Hum Genet. 2013;58(8):548-52.

11. Schielen P, Kemper EA, Gelb MH. Newborn screening for lysosomal storage diseases: a concise review of the literature on screening methods, therapeutic possibilities and regional programs. Int J Neonatal Screen. 2017. https://doi.org/10.3390/ ijns3020006.

12. Arends M, Hollak CE, Biegstraaten M. Quality of life in patients with Fabry disease: a systematic review of the literature. Orphanet J Rare Dis. 2015;10:77.

13. Waldek S, Patel MR, Banikazemi M, Lemay R, Lee P. Life expectancy and cause of death in males and females with Fabry disease: findings from the Fabry Registry. Genet Med. 2009;11(11):790-6.

14. Banikazemi M, Bultas J, Waldek S, Wilcox WR, Whitley $\mathrm{CB}$, McDonald $\mathrm{M}$, et al. Agalsidase-beta therapy for advanced Fabry disease: a randomized trial. Ann Intern Med. 2007;146(2):77-86.

15. Hoffmann B, Garcia de Lorenzo A, Mehta A, Beck $M$, Widmer U, Ricci R, et al. Effects of enzyme replacement therapy on pain and health related quality of life in patients with Fabry disease: data from FOS (Fabry Outcome Survey). J Med Genet. 2005;42(3):247-52.

16. Hughes DA, Elliott PM, Shah J, Zuckerman J, Coghlan G, Brookes J, et al. Effects of enzyme replacement therapy on the cardiomyopathy of Anderson-Fabry disease: a randomised, doubleblind, placebo-controlled clinical trial of agalsidase alfa. Heart. 2008;94(2):153-8.

17. Schiffmann R, Kopp JB, Austin HA 3rd, Sabnis S, Moore DF, Weibel T, et al. Enzyme replacement therapy in Fabry disease: a randomized controlled trial. JAMA. 2001;285(21):2743-9.

18. El Dib R, Gomaa H, Carvalho RP, Camargo SE, Bazan R, Barretti P, et al. Enzyme replacement therapy for Anderson-Fabry disease. Cochrane Database Syst Rev. 2016;7:CD006663.
19. Amicus Therapeutics, Inc. FDA Approves Galafold $^{\mathrm{TM}}$ (migalastat) for the Treatment of Certain Adult Patients with Fabry Disease. 2018. http://ir. amicusrx.com/news-releases/news-release-details/fdaapproves-galafoldtm-migalastat-treatment-certainadult. Accessed 21 Nov 2018.

20. European Medicines Agency. EPAR summary for the public. Galafold (migalastat). 2016. http://www. ema.europa.eu/docs/en_GB/document_library/EPAR_ -_Summary_for_the_public/human/004059/WC50 0208437.pdf. Accessed 27 Mar 2018.

21. Amicus Therapeutics UK Ltd. Galafold ${ }^{\mathrm{TM}}$ (migalastat) $123 \mathrm{mg}$ hard capsules. Summary of Product Characteristics. http://www.ema.europa.eu/docs/ en_GB/document_library/EPAR_-_Product_Infor mation/human/004059/WC500208434.pdf. Accessed 16 Apr 2018.

22. Welford RWD, Muhlemann A, Garzotti M, Rickert $\mathrm{V}$, Groenen PMA, Morand $\mathrm{O}$, et al. Glucosylceramide synthase inhibition with lucerastat lowers globotriaosylceramide and lysosome staining in cultured fibroblasts from Fabry patients with different mutation types. Hum Mol Genet. 2018;27(19):3392-403.

23. ClinicalTrials.gov. Efficacy and Safety of Lucerastat Oral Monotherapy in Adult Subjects With Fabry Disease (MODIFY). ClinicalTrials.gov identifier: NCT03425539. https:/clinicaltrials.gov/ct2/show/ NCT03425539. Accessed 06 Nov 2018.

24. Cleeland CS, Ryan KM. Pain assessment: global use of the Brief Pain Inventory. Ann Acad Med Singapore. 1994;23(2):129-38.

25. Politei JM, Bouhassira D, Germain DP, Goizet C, Guerrero-Sola A, Hilz MJ, et al. Pain in Fabry disease: practical recommendations for diagnosis and treatment. CNS Neurosci Ther. 2016;22(7):568-76.

26. Gibas AL, Klatt R, Johnson J, Clarke JT, Katz J. A survey of the pain experienced by males and females with Fabry disease. Pain Res Manag. 2006;11(3):185-92.

27. Sanchez MI, Bercik P. Epidemiology and burden of chronic constipation. Can J Gastroenterol. 2011;25(Suppl B):11B-5B.

28. Schmidt FM, Santos VL. Prevalence of constipation in the general adult population: an integrative review. J Wound Ostomy Continence Nurs. 2014;41(1):70-6.

29. Uceyler N, Ganendiran S, Kramer D, Sommer C. Characterization of pain in fabry disease. Clin J Pain. 2014;30(10):915-20. 
30. Fabry Registry. Annual Report 2013. 2013. http:// www.fabry.org/fsig.nsf/PDFs/PDFsR/\$File/2013_ Annual_Report.pdf. Accessed 8 Mar 2018.

31. Fabry Outcomes Survey. Annual Report 2016. 2017. http://www.fabrynetwork.org/wp-content/uploads/ 2017/11/FOS-Patient-Report-2016-Final.pdf. Accessed 8 Mar 2018.

32. Glass DC, Kelsall HL, Slegers C, Forbes AB, Loff B, Zion D, et al. A telephone survey of factors affecting willingness to participate in health research surveys. BMC Public Health. 2015;15:1017.
33. Peters FP, Vermeulen A, Kho TL. Anderson-Fabry's disease: alpha-galactosidase deficiency. Lancet. 2001;357(9250):138-40.

34. Lavalle L, Thomas AS, Beaton B, Ebrahim H, Reed $\mathrm{M}$, Ramaswami U, et al. Phenotype and biochemical heterogeneity in late onset Fabry disease defined by N215S mutation. PLoS ONE. 2018;13(4): e0193550. 